\title{
High Resolution Optical and Infrared Images of Circumstellar Disks
}

\author{
Deborah Padgett \\ MS 220-6, California Institute of Technology, Pasadena, CA 91125, \\ USA;dlp@ipac.caltech.edu \\ Karl Stapelfeldt \\ MS 183-900, Jet Propulsion Laboratory, California Institute of \\ Technology, Pasadena CA 91109, USA; krs@exoplanet.jpl.nasa.gov
}

\begin{abstract}
.
As the number of detected extrasolar planetary systems has steadily grown over the past five years, so too has the number of circumstellar disks with resolved images. In this contribution, we take stock of the current inventory of disk images in the optical and infrared; summarize the results of a new disk imaging survey conducted with the Hubble Space Telescope; review the major inferences that can be drawn about disk structure from the extant images; and suggest areas for future progress.
\end{abstract}

\section{Introduction and Background}

Circumstellar disks are the builders of worlds; they spin ordinary interstellar gas and dust into astronomical gold, the planetary systems which play host to life in the universe. In the first 10 million years of a star's life its dense natal disk dissipates, leaving behind a tenuous debris disk and, at least in some cases, a planetary system. In order to understand this key chapter in the story of our origins, astronomers seek detailed views of circumstellar disks. Remarkable advances in disk imaging science have taken place in the past decade, and the field is poised for further progress.

The first well-resolved image of a circumstellar disk was made in scattered light using optical coronagraphy in 1984 ( $\beta$ Pictoris; ref 36). Although it was expected that many more disk images would soon follow, optical imaging surveys did not succeed in detecting disks among other main sequence stars with infrared excess (35). The advent of the Hubble Space Telescope (HST) rejuvenated optical disk imaging $(3,5,16)$, and together with adaptive optics (AO) on large ground-based telescopes, has provided images of several young disks in the nearinfrared $(29,31,32,10 ; 7,8,27,30)$. Optical and near-infrared observations have provided the highest resolution images $\left(0.05^{\prime \prime}=7 \mathrm{AU} @ 140 \mathrm{pc}\right)$ of disks obtained to date. Finally, high-resolution thermal infrared imaging has begun to make important contributions to the field $(17,21,25,41)$.

Circumstellar disks are usually broken down into two major categories: dense, optically thick young disks associated with pre-main sequence objects 


\begin{tabular}{|c|c|c|c|c|}
\hline $\begin{array}{l}\text { Object } \\
\text { Name }\end{array}$ & $\begin{array}{c}\text { Scattered } \\
\text { Light }\end{array}$ & $\begin{array}{l}\text { Thermal } \\
\text { Infrared }\end{array}$ & $\begin{array}{l}\text { Radius } \\
\text { (AU) }\end{array}$ & $\begin{array}{c}\text { Recent } \\
\text { References }\end{array}$ \\
\hline$\epsilon$ Eridani & no & yes & 80 & 19,2 \\
\hline Fomalhaut & no & yes & 140 & 19,2 \\
\hline Vega & no & yes & 120 & 19,2 \\
\hline$\beta$ Pictoris & YES & YES & 1100 & $20,16,28$ \\
\hline HR 4796 & YES & YES & 80 & $31,17,21,41$ \\
\hline HD 141569 & YES & yes & 500 & 7 \\
\hline TW Hydrae & YES & no & 200 & 22,44 \\
\hline HD 100546 & YES & yes & 515 & 1,13 \\
\hline HD 163296 & YES & no & 450 & 12 \\
\hline AB Aurigae & yes & yes & 1320 & 24 \\
\hline DG Tauri B & yes & no & 300 & 29 \\
\hline GG Tauri & YES & no & 260 & $30,34,23$ \\
\hline GM Aurigae & YES & no & 300 & 37,32 \\
\hline HH 30 & YES & no & 315 & $5,10,37,39$ \\
\hline HK Tauri B & YES & yes & 105 & 25,40 \\
\hline HV Tauri $\mathrm{C}$ & YES & no & 50 & 27,38 \\
\hline Haro 6-5B & YES & no & 300 & 29 \\
\hline IRAS $04302+2247$ & YES & no & 500 & 29 \\
\hline UY Aurigae & YES & no & 520 & 9 \\
\hline 2MASSI J1628137-243139 & YES & no & 300 & 14 \\
\hline IRAS $04325+2402$ & YES & no & 30 & 15 \\
\hline IRAS $04158+2805$ & YES & no & 1100 & 26 \\
\hline OphE-MM3 & yes & no & $\leq 140$ & 4 \\
\hline CRBR 2422.8-3423 & yes & no & $\overline{\leq} 140$ & 4 \\
\hline $\mathrm{LkH} \alpha 263 \mathrm{C}$ & yes & no & 150 & 6,18 \\
\hline Orion $114-426$ & YES & no & 500 & 3 \\
\hline Orion $121-1925$ & yes & no & 185 & 3 \\
\hline Orion $132-1832$ & yes & no & 345 & 3 \\
\hline Orion $163-026$ & yes & no & 185 & 3 \\
\hline Orion $165-254$ & yes & no & 70 & 3 \\
\hline Orion $167-231$ & yes & no & 115 & 3 \\
\hline Orion $172-028$ & yes & no & 160 & 3 \\
\hline Orion $182-332$ & yes & no & 70 & 3 \\
\hline Orion $182-413$ & yes & no & 334 & 3 \\
\hline Orion $183-405$ & YES & no & 160 & 3 \\
\hline Orion 191-232 & yes & no & 70 & 3 \\
\hline Orion $203-506$ & yes & no & 90 & 3 \\
\hline Orion $218-354$ & YES & no & 320 & 3 \\
\hline Orion $218-529$ & yes & no & 90 & 3 \\
\hline Orion $239-334$ & yes & no & 115 & 3 \\
\hline Orion 294-606 & yes & no & 230 & 3 \\
\hline
\end{tabular}

Table 1. An inventory of resolved circumstellar disks. A 'YES' entry indicates that the disk is well-resolved at this wavelength; a 'yes' entry means the disk is marginally resolved, with only its outer radius, position angle, and inclination measured. Debris disks are listed in the top section, and young stellar object disks in the lower section. Sources are listed in order of heliocentric distance. An updated version of this table will be kept at http://exoplanet.jpl.nasa.gov/diskimcat. 


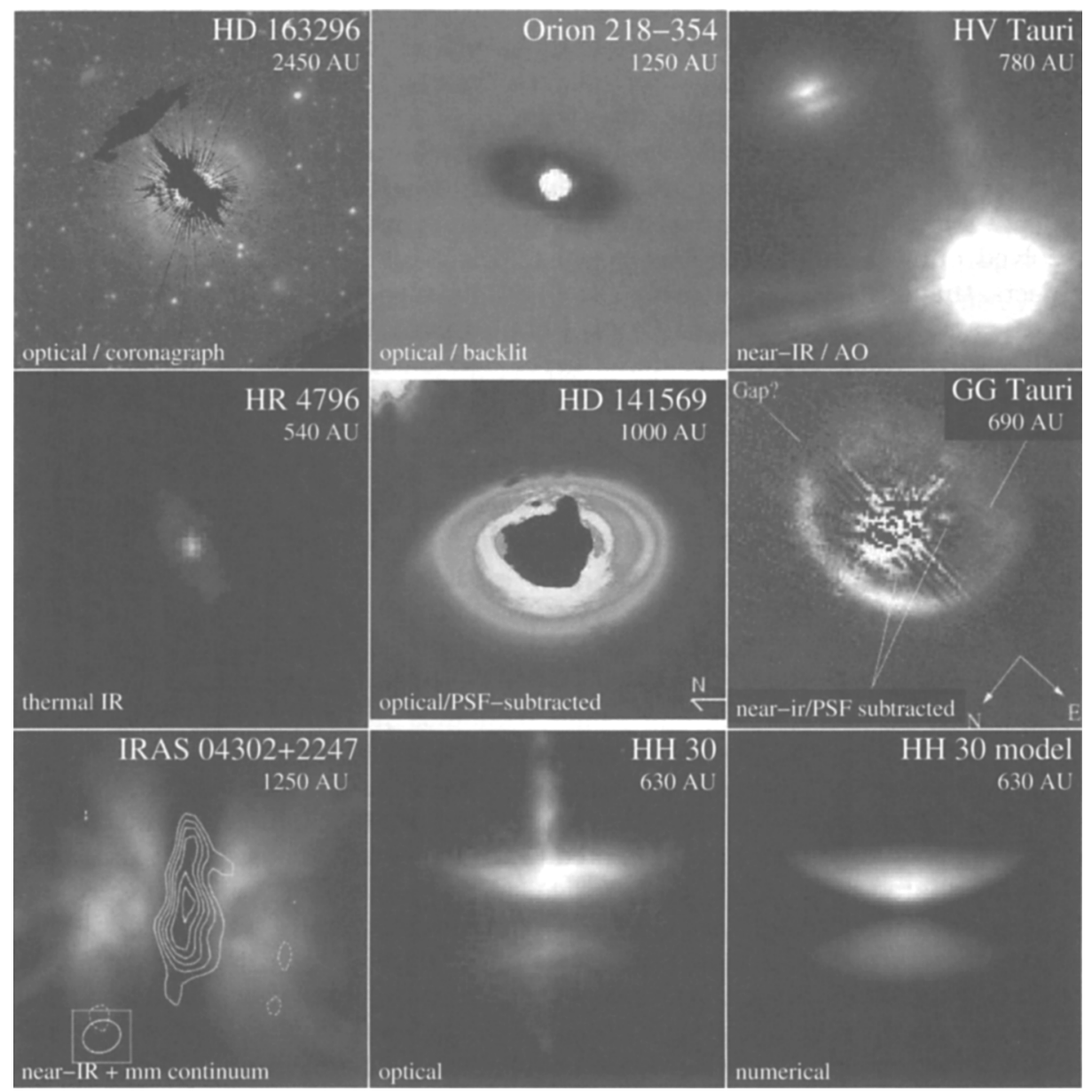

Figure 1. Images of circumstellar disks. Each panel is labeled with the object name, wavelength, and the size of the image field of view. From left to right in each row, the image sources are references top: (12), (3), (27); center: (21), (7), (34); and bottom: (29), (5), (5). 
such as T Tauri and Herbig Ae stars; and more tenuous, optically thin debris disks associated with nearby, much older main sequence stars. The young disks are gas rich, while the debris disks are dust-dominated; and correspondingly, the young disks have much larger masses comparable to the theoretical mass $(0.01$ $\mathrm{M}_{\odot}$ ) for the solar nebula which spawned our own Sun's planetary system. Small dust grains, similar to their ISM progenitors, are plentiful in the young disks; whereas in debris disks, larger second-generation grains derived from planetesimal collisions appear to dominate (2). As planet formation likely takes place during the evolutionary transition between the two kinds of disks, there is great interest in indentifying intermediate cases.

Table 1 lists the spatially resolved circumstellar disks known to the authors as of late 2003. Not listed are unpublished results, IR interferometry results,and objects for which the disk detection is in doubt, as well as objects which are resolved only at millimeter wavelengths. Disks are suspected for many more objects than listed in this table (such as main sequence stars with infrared excess, and externally ionized Orion Nebula "proplyds"); however, only those with resolved images are considered here. The current inventory of optical/IR imaged disks is at least 41 objects. Eighteen are well-resolved, with significant internal structure visible. There are 35 resolved disks associated with young stellar objects and only 6 resolved debris disks. Optical and near-IR imaging has produced the largest number of disk images, followed closely by $\mathrm{mm} / \mathrm{submm}$ techniques. A representative set of disk images is shown in Fig. 1.

\section{An HST Survey of Nearby T Tauri Disks}

Disk images at high spatial resolution and high image contrast can be made in optical and near-IR reflected light using the HST. The first deep imaging survey of T Tauri stars with HST, conducted with the WPFC2 camera in two snapshot observing programs, has just completed (37). The sample consisted of 153 young stars associated with the Taurus-Auriga, Chamaeleon, Lupus, Ophiuchus, and Corona Australis star-forming clouds at distances ranging from 140-160 pc. Most of the targets possess millimeter continuum emission indicating the presence of circumstellar disks, and/or optical polarization suggesting the presence of compact reflection nebulosity.

A wide variety of circumstellar nebulae have been found in this data set. More than a dozen sources show extended nebulosity reminiscent of circumstellar envelopes, with appearances ranging from symmetric bipolar cavities to irregular structures without obvious interpretation. Jet emission ranging from diffuse patches to compact knots is seen in 15 objects. Most interesting are eight sources where HST directly detects the disks in scattered light. These include the edgeon disks in the HK Tau (40) and HV Tau binary systems (38); the circumbinary ring in UY Aur; intermediate inclination disks associated with GM Aur, GO Tau, Sz 82, and DoAr 25; and a face-on disk around TW Hya (23). Modeling of the TW Hya images has found a rough order-of-magnitude agreement in disk mass estimates independently derived at optical and millimeter wavelengths. A similar agreement was also found in the case of HH 30 (5). This suggests that there has been no major depletion of micron-sized dust grains in the outer 
regions of these disks, and thus any planet formation process must still be in its earliest stage.

For two-thirds of the targets observed, however, HST/WFPC2 detects no nebulosity at all: only the stellar point spread function is seen. This includes 15 objects where millimeter interferometers have clearly resolved disks larger than 3 arcsec in diameter, and an additional 32 sources with millimeter continuum emission. Adaptive optics surveys have also failed to detect the scattered light from a similar but smaller sample of disks surveyed to date. A number of possibilities are available to explain why these disks are undetected in scattered light, including unfavorable orientation, a highly flattened geometry, and evolution in dust particle properties.

Finally, the number of edge-on disks detected in the survey was lower than expected. Geometrical arguments suggest that $\sim 15 \%$ of randomly oriented, optically thick disks should occult their central star, yet only 4/153 were found in this survey. Since an edge-on disk diminishes the total flux from the system by 3-5 mags, it is clear that existing $\mathrm{T}$ Tauri star catalogs suffer from a selection bias against these objects. It is likely that a dozen young edge-on disks remain to be identified in nearby molecular cloud complexes.

\section{Trends in Disk Structure}

Disk geometry, density, temperature distribution, and dust properties are best constrained through detailed comparisons of data with model images. Notable recent examples of this approach include multiwavelength image modeling of the GG Tau circumbinary ring (45); $\chi^{2}$-optimized model fitting to images of $\mathrm{HH}$ 30 's edge-on disk $(5,43,46)$; combined image and spectral energy distribution modeling of the HR 4796 debris disk (21); and self-consistent radiative transfer modeling of the resolved millimeter continuum, NIR scattered light, and IRAS SED of IRAS $04302+2247$ (47) Key results of these and other efforts can be broken down in the following way:

Disk Radial Structure. All of the resolved disks have a radial extent comparable to or larger than our solar system's Kuiper Belt. In several cases, outer radii extending beyond $500 \mathrm{AU}$ have been found $(12,20,29)$. Central holes cleared by tidal interactions have been directly imaged in young circumbinary disks $(30,8,34)$. They have also been imaged in debris disks; however, it is not known if the central dust clearing in these cases has been accomplished by dynamical or radiative processes. Several debris disks have noteworthy radial structure: HR 4796 has been resolved as a relatively narrow ring (31), while HD 141569 has a prominent radial gap $(31,7)$. Radial density gradients have proven difficult to infer in optically thick young disks. WFPC2 and NICMOS imaging of the face-on disk around TW Hya find that the surface brightness profile changes with radius, forming annular zones $(23,34)$. STIS coronagraphy of HD 163296, an isolated Ae star at $122 \mathrm{pc}$, show that its $450 \mathrm{AU}$ disk has an inner zone of reduced scattering (12). The disk with the best-determined radial density gradient is that of $\beta$ Pic, where a $\mathrm{r}^{-3}$ surface density law is indicated by the scattered light brightness profile $(20,16)$.

Disk Vertical Structure. Vertical profiles can be studied in edge-on disk systems. Flaring (i.e., increasing disk thickness with radius) is now directly ob- 
served in young stellar object disks. Detailed studies of the HH 30 disk indicate a Gaussian vertical profile with a scale height comparable to that expected from local thermal equilibrium $(5,43)$. However, a smaller than expected scale height has been measured in the disk of HK Tau B (40), possibly indicating that the dust has settled with respect to the gas in this disk. In debris disks, where gas is largely absent, the disk vertical profile traces the orbital inclination distribution for the parent bodies whose collisions are replenishing the dust population. Conversely, the disk of $\beta$ Pic is especially thin, with an approximately exponential vertical profile, and a warped inner region which may indicate the presence of a perturbing body near its inner edge (28).

Disk Asymmetries. Azimuthal asymmetries cannot persist in a disk for more than a few orbital timescales before being disrupted by Keplerian shear. Therefore, the presence of such asymmetries strongly suggests that a disk is being perturbed by some other object. The $\beta$ Pic disk has several subtle asymmetries such as differences in its radial brightness profile and vertical thickness between the NE and SW extensions (20); one possible explanation is that the disk was recently disturbed by the close passage of an as-yet-unidentified $\mathrm{M}$ dwarf star. The ansae of the HR 4796 ring appear slightly asymmetric in both scattered light and thermal IR imaging $(41,31)$. Finally, a most unusual variable brightness asymmetry has been observed in optical images of $\mathrm{HH}$ 30: a bright spot of light appears to be moving around the disk (39). While this may be produced by stellar hotspot illumination, another viable model is beaming/shadowing of the outer disk as starlight propagates through azimuthal inhomogeneities in the optically thick inner disk.

Grain Growth. One of the more contentious conclusions derived from modeling of disk images is that dust properties in some disks are significantly different from those of interstellar grains. Disks for which grain growth is claimed include HH 30 (10), HK Tauri B $(11,25)$, and Orion 114-426 $(42,33)$. In these cases, the interpretation of grain growth depends on the variation of disk feature sizes with wavelength. For example, Throop et al. (42) interpret the lack of significant size variation in the Orion 114-426 silhouette disk between $\mathrm{H} \alpha$ and $\mathrm{Pa} \alpha$ as evidence for large grains. The fact that the silhouette does apparently become smaller in $4 \mu \mathrm{m}$ images constrains the grain size to no larger than 2 - $3 \mu \mathrm{m}$ (33). IRAS $04302+2247$ system provides evidence that the properties of grains in the envelope may be more ISM-like than the grains in the disk (47). However, many of these analyses depend on assumptions made about disk parameters which may be degenerate with grain optical properties, such as the radial density profile. Multiparameter image fitting may be the best technique for determining the robustness of conclusions regarding disk grain sizes (43).

\section{Future Prospects}

There is much room for growth in the field of disk imaging science in the coming decade. Specific activities that would advance the field include: 1) Expand the inventory of disks imaged at all wavelengths and in all stellar contexts. New debris disk images are especially needed, as only six of these objects have been resolved to date. 2) Make greater efforts to derive disk structure parameters by comparing models to multiwavelength resolved images and spectral energy 
distributions. Detailed modeling work has not kept pace with the growing number of well-resolved disk images. 3) Map the radial gradients in gas chemistry and dust properties within disks, to document the evolution from interstellar to planetary material. Imaging evidence of dust settling to a disk's midplane should be obtainable in young edge-on disks. 4) Establish the presence and distribution of dust in our own Kuiper Belt, for direct comparison to external debris disk systems. Perhaps the ultimate goal is 5) To locate planets within resolved disks and relate them to disk radial structures and asymmetries.

To accomplish these goals, improvements will be needed in observational methods at all wavelengths. In scattered light and thermal infrared images, the most tenuous disk reliably imaged to date is still that of $\beta$ Pic - with $10^{4}$ times the vertical optical depth of our solar system's zodiacal light. In the optical and nearinfrared, detection of more tenuous disks will require much better techniques for high contrast imaging. In the thermal infrared, the chief problem is the limited sensitivity to low disk surface brightnesses when observing through atmospheric sky backgrounds. Improvements here will require large telescopes in space, and nulling interferometers on the ground.

Fortunately there are upcoming astronomical projects and facilities that will offer significant benefits to disk imaging science. One of these is NASA's Space Infrared Telescope Facility (SIRTF), launched in August 2003. Even with its modest $0.85 \mathrm{~m}$ aperture, SIRTF will be able to detect and resolve dozens of nearby debris disks at $70 \mu \mathrm{m}$, down to optical depths less than 100 times the solar system's zodiacal light. Furthermore, SIRTF should photometrically detect thousands of new disks suitable for follow-up imaging studies. Particularly interesting will be nearby late-type stars possessing debris disks as optically thick as that of $\beta$ Pic, but which went undetected by IRAS due to the central stars' low luminosities.

In the optical and near-infrared, it does not appear that the James Webb Space Telescope (JWST) or new AO systems on very large telescopes will offer major image contrast improvements. Current adaptive optics systems correct the wavefront to only $\lambda / 15$ or so, while HST has fixed wavefront errors of $\sim \lambda / 40$. These errors lead to unwanted scattering of light into a halo around the central star. Increases in aperture size, or in the number of actuators used to control the wavefront, yield only small improvements in image contrast. The key (and often unappreciated) requirement is precise control of the optical wavefront: errors must be reduced toward $\sim \lambda / 1000$ at the critical mid-spatial frequencies to enable direct imaging of exozodiacal dust at levels comparable to that of our own solar system. This goal will soon be achievable using a dedicated space telescope intrumented with a precision deformable mirror and a coronagraph. Absent this, only incremental progress can be expected in optical and nearinfrared disk imaging in the coming decade.

\section{References}

(1) Augereau, A., Lagrange, A., Mouillet, D., and Ménard, F. 2001, A.\&A., 365, 78.

(2) Backman, D. and Paresce, F. 1993, in Protostars \& Planets III, E. Levy and J. Lunine eds., Univ. of Arizona Press, Tuscon, pp. 1253-1304.

(3) Bally, J., O'Dell, C.R., and McCaughrean, M.J. 2000, A.J., 119, 2919.

(4) Brandner, W. et al. 2003, A\&A 364, L13. 
(5) Burrows, C.J. et al. 1996, Ap.J., 473, 437.

(6) Chauvin, G. et al. 2002, A\&A 394, 949.

(7) Clampin, M. et al. 2003, A.J. 126, 385.

(8) Close, L.M. et al. 1998, Ap.J., 499, 883.

(9) Close, L.M. et al. 1997, Ap.J., 478, 766.

(10) Cotera, A. et al. 2001, Ap.J. 556, 958.

(11) Duchene, G. et al. 2003, A.\& A. 400, 559.

(12) Grady, C.A. et al. 2000, Ap.J., 544, 895.

(13) Grady, C.A. et al. 2001, A.J. 122, 3396.

(14) Grosso, N. et al. 2003, Ap.J. 586, 296

(15) Hartmann, L. et al. 1999, A.J. 118, 1784.

(16) Heap, S.R. et al. 2000, Ap.J., 539, 435.

(17) Jayawardhana, R. et al. 1998, Ap.J., 503, L79.

(18) Jayawardhana, R. et al. 2002, Ap.J. 571, L51.

(19) Kalas, P. and Jewitt, D. 1996, A.J., 111, 1347.

(20) Kalas, P., and Jewitt, D. 1995, A.J., 110, 794.

(21) Koerner, D., Ressler, M., Werner, M., and Backman, D. 1998, Ap.J., 503, L83.

(22) Krist, J., Stapelfeldt, K., Ménard, F., Padgett, D., Burrows, C. 2000, Ap.J., 538, 793.

(23) Krist, J. et al. 2002, Ap.J. 570, 785.

(24) Marsh, K. et al. 1995, Ap.J., 451, 777.

(25) McCabe, C., Duchene, G., \& Ghez, A.M. 2003, Ap.J. 588, 113

(26) Menard, F. et al. 2003, A.\& A., in press

(27) Monin, J.-L., and Bouvier, J. 2000, A.\&A., 356, L75.

(28) Mouillet, D., Larwood, J., Papaloizou, J., Lagrange, A. 1007 M.N.R.A.S., 292, 896.

(29) Padgett, D.L., Brandner, W., Stapelfeldt, K.R., Strom, S.E., Terebey, S., Koerner, D. 1999, A.J., 117, 1490.

(30) Roddier, C., Roddier, F., Northcott, M., Graves, J., Jim, K. 1996, Ap.J., 463, 326.

(31) Schneider, G. et al. 1999, Ap.J. 513, L127

(32) Schneider, G. et al. 2003, A.J. 125, 1467.

(33) Shuping, R. Y. et al. 2003, Ap.J. 587, 109

(34) Silber, J., Gledhill, T., Duchêne, G., Ménard, F. 2000, Ap.J., 536, L89.

(35) Smith, B., Fountain, J., and Terrile, R. 1992, A.\&A., 261, 499.

(36) Smith, B. and Terrile, R. 1984, Science, 226, 1421.

(37) Stapelfeldt, K. et al. 2004 in preparation.

(38) Stapelfeldt, K. et al. 2003, Ap.J. 589, 410

(39) Stapelfeldt, K. et al. 1999, Ap.J., 516, L95.

(40) Stapelfeldt, K., Krist, J., Ménard, F., Bouvier, J., Padgett, D., Burrows, C. 1998, Ap.J., 502, L65.

(41) Telesco, C.M. et al. 2000, Ap.J. 530, 329.

(42) Throop, H. B. et al. 2001, Sci. 292, 1686.

(43) Watson, A, Stapelfeldt, K. 2003, in preparation

(44) Weinberger, A. et al. 2002, Ap.J. 566, 409.

(45) Wood, K., Crosas, M., and Ghez, A. 1999, Ap.J., 516, 335.

(46) Wood, K., Kenyon, S., Whitney, B., and Turnbull, M. 1998, Ap.J., 497, 404.

(47) Wolf, S., Padgett, D., \& Stapelfeldt, K. et al. 2003, Ap.J. 588, 373. 